\title{
Finite-Time Switched Second-Order Sliding-Mode Control of Nonholonomic Wheeled Mobile Robot Systems
}

\author{
Hao Ce, Wang Hongbin (iD, Cheng Xiaoyan (D), Zhou Zhen (iD, \\ Ge Shungang, and Hu Zhongquan \\ Key Lab of Industrial Computer Control Engineering of Hebei Province, Yanshan University, Qinhuangdao 066004, China \\ Correspondence should be addressed to Wang Hongbin; hb_wang@ysu.edu.cn
}

Received 26 November 2017; Accepted 12 March 2018; Published 9 May 2018

Academic Editor: Eloy Irigoyen

Copyright (c) 2018 Hao Ce et al. This is an open access article distributed under the Creative Commons Attribution License, which permits unrestricted use, distribution, and reproduction in any medium, provided the original work is properly cited.

\begin{abstract}
A continuous finite-time robust control method for the trajectory tracking control of a nonholonomic wheeled mobile robot (NWMR) is presented in this paper. The proposed approach is composed of conventional sliding-mode control (SMC) in the internal loop and modified switched second-order sliding-mode (S-SOSM) control in the external loop. Sliding-mode controller is equivalently represented as stabilization of the nominal system without uncertainties. An S-SOSM control algorithm is employed to counteract the impact of state-dependent unmodeled dynamics and time-varying external disturbances, and the unexpected chattering has been attenuated significantly. Particularly, state-space partitioning is constructed to obtain the bounds of uncertainty terms and accomplish different control objectives under different requirements. Simulation and experiment results are used to demonstrate the effectiveness and applicability of the proposed approach.
\end{abstract}

\section{Introduction}

In the past few decades, the design of a robust control of nonholonomic wheeled mobile robot (NWMR) has been a difficult task due to the nonlinearities, nonholonomic constraints, and uncertainties in the system [1]. So, it received wide attention and became a topic of great research interest due to the practicability of its application [2]. Basically, trajectory tracking is one of the important motion control problems, which not only requires a designed controller [3] but also has to robustly stabilize the nonlinear system against the system uncertainties. Depending on whether the NWMR is described by kinematics $[4,5]$ or dynamics $[6,7]$, the tracking problem can be classified as either a kinematic tracking problem or a dynamic tracking problem. However, it is hard to obtain excellent tracking performance if just a kinematic model is considered, because of the existence of errors between the actual velocity and the output of the controller. It is more realistic to consider the tracking problem of both dynamic and kinematic models $[8,9]$ than that of the kinematic model, where only two control signals are needed for the robot under the kinematic constraints.
Several approaches have been proposed and numerous valuable results for dynamic trajectory tracking control problems have been obtained. The main control approaches include backstepping approach [10], neural network control [11, 12], sliding-mode control (SMC) [13], fuzzy algorithm [14], and adaptive approach $[15,16]$. The SMC, a robust control technique which has many attractive features such as robustness to parameter variation and insensitivity to disturbance, is a suitable control method for NWMR tracking control [17]. In comparison with other robust control approaches mentioned above, it has a simple control structure and can provide fast dynamic responses. A number of applications can be found in the literature [18]. Considering the system uncertainties and external disturbances, a hierarchical sliding-mode underactuated controller [19] is proposed for the system with completely and partially known frictions and uncertainties. Focusing on the fact that the SMC method has high robustness, the tracking control for a NWMR with unknown system uncertainties was solved by the method [20, 21]. However, conventional sliding-mode controllers feature an obvious drawback where control torque is discontinuous and 
oscillates at a high frequency, which cannot be tolerated in some practical applications.

To avoid this phenomenon, some methods have been proposed, such as equivalent control, boundary layer method [22], and intelligent SMC [23]. A second-order sliding-mode (SOSM) controller [24-26], which is effective in extending the performance and robustness of the standard slidingmode controller, was also proposed to reduce the chattering effect $[27,28]$. However, the system uncertainties and external disturbance are not completely considered and the bounds of uncertainties are assumed to be known, which would limit the wide application of the designed control method. Aiming at the above problems, the PD-SOSM controller was proposed to deal with the NWMR [29] with system uncertainties and disturbance. An adaptive second-order terminal slidingmode controller [30] was proposed, and the bounds of unknown parameters were estimated using the adaptation law, but the estimate progress is rather complicated. Meanwhile, it is also important to note that most of the tracking problems treated in the literature have been mainly concerned with NWMR whose trajectory converges to the desired trajectory asymptotically. However, the tracking control achieved in finite time is required in some practical situations. It can be observed from the literature that the finite time is mainly achieved by the sign function of states [31] or relative states [32] for the nonlinear systems. Unlike most of the methods mentioned above, state-space partitioning $[33,34]$ is embedded into the switched second-order sliding-mode (SSOSM) controllers, such that the trajectories can be driven onto the desire trajectory in finite time and do not require the knowledge of the bounds of the uncertainties. But it is mainly applied to the systems whose trajectories converge to a constant value of zeros instead of time-varying desired trajectories, which is a big limitation of the application. In this paper, the SOSM controller is adopted in the external loop, overcoming the disturbance and uncertainties without obtaining the upper bounds in advance.

Motivated by the idea [21, 33, 34], we further propose a combination of an integral sliding-mode controller and an SSOSM controller. At first, an integral sliding-mode controller is applied to the system without uncertainties. Then, an SSOSM algorithm is employed to conquer the unmodeled dynamics and external disturbances and guarantee system's precision and robustness. In the end, comparative simulation and experimental studies demonstrate the effectiveness and applicability of the proposed approach.

The paper is organized as follows. Section 2 gives the NWMR's kinematic and dynamic models and the SMC algorithm. In Section 3, the improved S-SOSM algorithm is proposed. In Section 4, the closed-loop stability and convergence are given to demonstrate the efficiency and the correctness of the improvement algorithm. In Section 5, the simulation and experimental results are provided, and the conclusions are given in Section 6.

\section{SMC for NWMR}

2.1. Description of NWMR Systems. The NWMR shown in Figure 1 is an example of a nonholonomic mechanical system

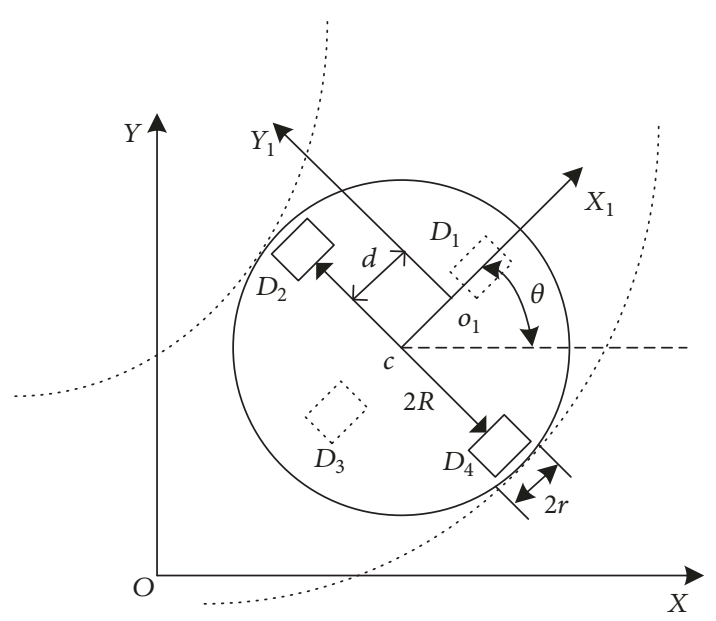

FIGURE 1: NWMR configuration.

consisting of a cart with two driving wheels (marked as $D_{2}$ and $D_{4}$ ) and two omnidirectional castors (marked as $D_{1}$ and $D_{3}$ ). It generally moves in the flat ground, so it is assumed that there is no gravitational term.

The dynamic and kinematic models of NWMR are adopted from [35]:

$$
\begin{aligned}
\bar{M}_{0}(q) \dot{\eta}+\bar{\tau}_{d}+\bar{F}(q, \dot{q}, \ddot{q}) & =\bar{B}(q) \tau, \\
\dot{q} & =S(q) \eta,
\end{aligned}
$$

where $\eta(t)=\left[v_{o 1}, w_{o 1}\right]^{\mathrm{T}}$ are the velocities of the robot, $v_{o 1}$ and $w_{o 1}$ denote the linear and angular velocities, $\bar{M}(q)=\bar{M}_{0}(q)+$ $\Delta \bar{M}(q) \in \mathbb{R}^{2 \times 2}$ is a symmetric positive-definite inertia matrix, $\bar{\tau}_{d} \in \mathbb{R}^{2 \times 1}$ denotes bounded external disturbances, $\bar{F}(q, \dot{q}, \ddot{q})=\Delta \bar{M}(q) \dot{\eta} \in \mathbb{R}^{2 \times 1}$ is the unmodeled dynamics, $\bar{B}(q) \in \mathbb{R}^{2 \times 2}$ is the vector of transformation matrix, and $q=[x, y, \theta]^{\mathrm{T}}$ with associated velocities $\dot{q}$ and acceleration $\ddot{q}$ are controlled by applied torque $\tau \in \mathbb{R}^{2 \times 1}$. Moreover,

$$
\begin{aligned}
S(q) & =\left[\begin{array}{cc}
\cos \theta & -d \sin \theta \\
\sin \theta & d \cos \theta \\
0 & 1
\end{array}\right], \\
\bar{M}_{0}(q) & =\left[\begin{array}{cc}
m & 0 \\
0 & I-m d^{2}
\end{array}\right], \\
\bar{B}(q) & =\frac{1}{r}\left[\begin{array}{cc}
1 & 1 \\
R & -R
\end{array}\right], \\
\tau & =\left[\begin{array}{c}
\tau_{r} \\
\tau_{l}
\end{array}\right],
\end{aligned}
$$

where $m$ and $I$ are the mass and inertia of the NWMR; $d$ is the distance between points $O_{1}$ and $c ; r$ and $2 R$ represent the radius of the wheel and the distance of the two driving wheels, respectively. 
2.2. Sliding-Mode Function and Controller Design. The nominal system is governed using the sliding-mode controller, which can effectively realize the tracking objective of the system without uncertainties.

The tracking errors are defined as

$$
q_{e}=\left[\begin{array}{lll}
e_{1} & e_{2} & e_{3}
\end{array}\right]^{\mathrm{T}}=T \tilde{q},
$$

with

$$
\begin{aligned}
& T=\left[\begin{array}{ccc}
\cos \theta & \sin \theta & 0 \\
-\sin \theta & \cos \theta & 0 \\
0 & 0 & 1
\end{array}\right], \\
& \tilde{q}=\left[\begin{array}{l}
x_{r}-x \\
y_{r}-y \\
\theta_{r}-\theta
\end{array}\right],
\end{aligned}
$$

where $q_{r}=\left[x_{r}, y_{r}, \theta_{r}\right]^{\mathrm{T}}$ denotes the posture of the reference NWMR.

An auxiliary velocity control input $\eta_{c}$ based on backstepping method [36] is given as

$$
\eta_{c}(t)=\left[\begin{array}{c}
v_{c} \\
w_{c}
\end{array}\right]=\left[\begin{array}{c}
v_{o 1 r} \cos e_{3}+k_{1} e_{1} \\
w_{o 1 r}+k_{2} v_{o 1 r} e_{2}+k_{3} v_{o 1 r} \sin e_{3}
\end{array}\right],
$$

where $v_{c}$ is the speed tracking controller output of linear velocity and $w_{c}$ is the angular velocity and $k_{i}>0, i=1,2,3$.

The integral sliding-mode surface is defined as

$$
s(t)=e_{c}(t)+\lambda \int_{0}^{t} e_{c}(\tau) d \tau
$$

where $e_{c}(t)=\eta(t)-\eta_{c}(t)$ and $\lambda$ is a positive surface integral constant.

If $e_{c}(t)$ moves to sliding-mode manifold and stays on it all the time, then it will be steered to the origin and the exponential stability is guaranteed. Considering $\dot{\boldsymbol{s}}(t)=0$ and using the model of the robot (1), the equivalent control law is designed as

$$
\tau_{e q}=\left(\bar{M}_{0}^{-1}(q) \bar{B}(q)\right)^{-1}\left(\dot{\eta}_{c}(t)-\lambda e_{c}(t)\right)
$$

The whole control input vector is assumed as follows:

$$
\tau=\tau_{e q}+\tau_{s w}
$$

where $\tau_{s w}$ is designed by guaranteeing the global stability of the NWMR against the unmodeled dynamics and external disturbances.

Substituting (8) and (9) into system (1) results in the following closed-loop unknown subsystem dynamics equation:

$$
\begin{aligned}
\dot{\eta}= & \bar{M}_{0}^{-1}(q) \bar{B}(q) \tau_{s w}-\lambda e_{c}(t) \\
& -\bar{M}_{0}^{-1}(q)\left(\bar{\tau}_{d}+\bar{F}(q, \dot{q}, \ddot{q})\right)+\dot{\eta}_{c}(t) .
\end{aligned}
$$

\section{S-SOSM Control}

In order to effectively deal with the trajectory tracking control problem for NWMR with state-dependent unmodeled dynamics and time-varying external disturbances, the definition of intermediate variables $z_{1}$ on the sliding-mode surface is employed.

Consider the second-order uncertain nonlinear auxiliary system

$$
\begin{aligned}
& \dot{z}_{1}=z_{2}, \\
& \dot{z}_{2}=f(z(t))+g(z(t)) v+h(t),
\end{aligned}
$$

where $z_{i}=\left[z_{i 1}, z_{i 2}\right], i=1,2, z=\left[z_{1}, z_{2}\right]^{\mathrm{T}} \in \mathbb{R}^{2}$ are auxiliary system state variables. $z_{1}=s(t)$ is the sliding variable. $f(z(t))$ and $g(z(t))$ are uncertain smooth functions that can be obtained from (7)-(10). $v$ is the time derivative of $\tau_{s w} . h(t)$ are time-varying external disturbances for which an upper bound $H$ is known.

To achieve the tracking objective, we need the following assumptions.

Assumption 1. $|f(z(t))| \leq F, 0<G_{1} \leq g(z(t)) \leq G_{2}$, where $F, G_{1}$, and $G_{2}$ are state-dependent positive constants.

Assumption 2. The extremal value $z_{\mathrm{Max}}$ of $z_{1}$ can be detected.

Define the state-space partitioning as follows.

Assume that the system state space $Z$ is divided into $n$ regions $R_{i}, i=1,2, \ldots, n$. The innermost region is defined as

$$
R_{i}:=\left\{\left(z_{1}, z_{2}\right):\left|z_{1}\right| \leq \bar{z}_{1, i},\left|z_{2}\right| \leq \bar{z}_{2, i}\right\}
$$

with $\bar{z}_{j, i+1}<\bar{z}_{j, i}, i=1,2, \ldots, n-1, j=1,2$. The outermost region is defined as

$$
R_{1}:=\left\{\left(z_{1}, z_{2}\right):\left|z_{1}\right|>\bar{z}_{1,1},\left|z_{2}\right|>\bar{z}_{2,1}\right\}
$$

where $Z_{i}=R_{i} / R_{i+1}, i=1,2, \ldots, n-1, Z_{n}=R_{n} . W_{i}=$ $\delta R_{i+1}, \quad i=1,2, \ldots, n-1$, denotes the edge of region $R_{i+1}$ (see Figure 2).

Conclusion 3. Under Assumption 1 and the description of the state-space partitioning in (12) and (13), the bounds of the uncertainty terms $f(z(t))$ and $g(z(t))$ can be determined based on the fact that the state variables of each region are bounded. One can rewrite

$$
\begin{aligned}
& \quad|f(z(t))| \leq F_{i}, \\
& \quad 0<g_{1, i} \leq g(z(t)) \leq g_{2, i}, i=1,2, \ldots, n .
\end{aligned}
$$




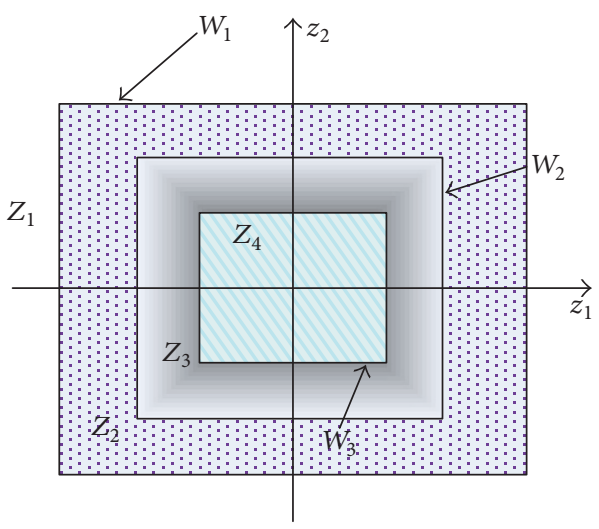

FIgURE 2: An example of the state-space partitioning.

Remark 4. In this paper, the state-space partitioning is employed to obtain the bounds of the uncertainty terms, so that the assumption of bound for the uncertain functions can be relaxed. Moreover, different from the traditional analysis methods such as Lyapunov functions [37, 38], it is difficult to build a Lyapunov function for the system, and it may be reconstructed if the system changes. It is noted that invariant sets are introduced in this paper, which is the key to prove that the trajectory converges to the desired trajectory. There is no need to build complex functions, and this will not change much with the system changes.

A set is expressed as

$$
\widetilde{R}_{i}=Z_{i} \backslash\left\{\overline{p_{1, i} p_{2, i}} \cup \overline{p_{5, i} p_{6, i}}\right\}, \quad i=1,2, \ldots, n,
$$

where

$$
\begin{aligned}
& \overline{p_{1, i} p_{2, i}} \\
& =\left\{\left(z_{1}, z_{2}\right) \in W_{i}: z_{1} \in\left[-z_{1, i}+\frac{z_{2}\left|z_{2}\right|}{2 \widetilde{V}_{i}}, z_{2}=\bar{z}_{2, i}\right]\right\} . \\
& \overline{p_{5, i} p_{6, i}} \\
& =\left\{\left(z_{1}, z_{2}\right) \in W_{i}: z_{1} \in\left[z_{1, i}-\frac{z_{2}\left|z_{2}\right|}{2 \widetilde{V}_{i}}, z_{2}=-\bar{z}_{2, i}\right]\right\} .
\end{aligned}
$$

If $z \in \widetilde{R}_{i}, i=1,2, \ldots, n$, the control law $v_{i}$ is defined as

$$
\begin{aligned}
& v_{i}=-\alpha_{i} V_{i} \operatorname{sign}\left(z_{1}-\beta z_{\mathrm{Max}}\right), \\
& \alpha_{i}= \begin{cases}\alpha_{i}^{*} & \left(z_{1}-\beta z_{\mathrm{Max}}\right)\left(z_{\mathrm{Max}}-z_{1}\right)>0 \\
1 & \text { else, }\end{cases}
\end{aligned}
$$

where $V_{i}$ is the control gain for the region $R_{i}, \alpha_{i}$ is the modulation factor, and $z_{\mathrm{Max}}$ is a piecewise constant function $\left(z_{\mathrm{Max}}=\right.$ $\left.z_{1}\left(t_{1}\right), z_{2}\left(t_{1}\right)=0\right), \beta=1 / 2$. The following constraints are satisfied to make the system trajectory converge to the desired trajectory in finite time:

$$
\begin{aligned}
& \alpha_{i}^{*} \in\left(\begin{array}{ll}
0 & 1
\end{array}\right] \cap\left(\begin{array}{ll}
0 & \frac{3 g_{1, i}}{g_{2, i}}
\end{array}\right), \\
& V_{i}>\max \left\{\begin{array}{ll}
\frac{F_{i}+H}{\alpha_{i}^{*} g_{1, i}} & \frac{4\left(F_{i}+H\right)}{3 g_{1, i}-\alpha_{i}^{*} g_{2, i}}
\end{array}\right\}, \\
& V_{i}>V_{\mathrm{Max}}=\max _{j=2,3, \ldots, n}\left(\frac{F_{j}+H}{\alpha_{j}^{*} g_{1, j}}\right),
\end{aligned}
$$

where $\widetilde{V}_{i}=\alpha_{i}^{*} g_{1, i} V_{\mathrm{Max}}-F_{i}-H>0, i=1,2, \ldots, n$.

If $z \in\left\{\overline{p_{1, i} p_{2, i}} \cup \overline{p_{5, i} p_{6, i}}\right\}, i=1,2, \ldots, n$, the control law $v$ is defined as

$$
\begin{aligned}
v_{i} & =-\alpha_{i} V_{i} \operatorname{sign}\left(z_{1}-\beta z_{\mathrm{Max}}\right), \\
v_{i-1} & =\alpha_{i} V_{i} \operatorname{sign}\left(z_{1}-\beta z_{\mathrm{Max}}\right),
\end{aligned}
$$

where $\alpha_{i}$ is chosen as (18).

Inserting (8) and (17)-(21) into (9), (9) can be rewritten as

$$
\tau_{i}=\left(\bar{M}_{0}^{-1}(q) \bar{B}(q)\right)^{-1}\left(\dot{\eta}_{c}(t)-\lambda e_{c}(t)\right)+\int v_{i} d t .
$$

Remark 5. The S-SOSM controller is applied into the design of the switching controller $\tau_{s w}$. The discontinuous sign function is replaced by a continuous function, so the unexpected chattering can be attenuated significantly.

Remark 6. Different control objectives in the different regions could be accomplished, for the curvature of the trajectory can be adjusted, for example, a short settling time or a minimal overshoot. There is no need to design different control strategies for the various control objects in different environments; for example, the UAV needs a minimal overshoot before reaching the target position and needs a short settling time when hovering in the target position.

\section{Convergence Analysis}

Theorem 7. For the second-order uncertain nonlinear auxiliary system, under the proposed controller, the following contraction property is featured:

$$
\left|z_{\operatorname{Max}_{i+1}}\right|<\left|z_{\operatorname{Max}_{i}}\right|, \quad i=1,2, \ldots, n-1 .
$$

Proof. Assume that $z_{1}(0)=z_{\mathrm{Max}}, z_{2}(0)=0$, and the initial point lies on the positive direction of the abscissa with the control law $v_{i}=-\alpha_{i} V_{i}$. When the commutation takes place at time instant $t_{2}$ such that $z_{1}\left(t_{2}\right)=z_{\mathrm{Max}} / 2$, the value of $z_{2}\left(t_{2}\right)$ becomes within the interval

$$
\begin{aligned}
& z_{2}\left(t_{2}\right) \\
& \in\left[-\sqrt{z_{\mathrm{Max}}\left(\alpha_{i}^{*} g_{2, i} V_{i}+F_{i}+H\right)}-\sqrt{z_{\mathrm{Max}}\left(\alpha_{i}^{*} g_{1, i} V_{i}-F_{i}-H\right)}\right],
\end{aligned}
$$

and when $t>t_{2}$, the auxiliary system with control law $v_{i}=$ $V_{i}$ shows that the trajectories intersect with the axis, and the intersection point belongs to the interval 


$$
\left[-\frac{\left(\alpha_{i}^{*} g_{2, i}-g_{1, i}\right) V_{i}+2\left(F_{i}+H\right)}{2\left(g_{1, i} V_{i}-F_{i}-H\right)} z_{\operatorname{Max}} \frac{\left(g_{2, i}-\alpha_{i}^{*} g_{1, i}\right) V_{i}+2\left(F_{i}+H\right)}{2\left(g_{2, i} V_{i}+F_{i}+H\right)} z_{\text {Max }}\right] .
$$

It is obvious that the right extreme of the interval is closer to the origin than the last intersection. Then, the sufficient condition for satisfying the contraction property is that the magnitude of the left extreme of the interval is less than $z_{\mathrm{Max}}$, which can be denoted by the following system of inequalities:

$$
\begin{aligned}
\alpha_{i}^{*} & \leq 1, \\
\alpha_{i}^{*} g_{1, i} V_{i} & \geq F_{i}+H, \\
\frac{\left(\left(\alpha_{i}^{*} g_{2, i}-g_{1, i}\right) V_{i}+2\left(F_{i}+H\right)\right)}{\left(g_{1, i} V_{i}-F_{i}-H\right)} & \leq 2 .
\end{aligned}
$$

Finally, it can be obtained that

$$
V_{i}>\max \left\{\frac{F_{i}+H}{\alpha_{i}^{*} g_{1, i}} \frac{4\left(F_{i}+H\right)}{3 g_{1, i}-\alpha_{i}^{*} g_{2, i}}\right\} .
$$

The proof process is the same when the initial point is at other locations.

For the second-order uncertain nonlinear auxiliary system, the control law $v$ steers the state variables to the origin.

Define

$$
\bar{R}_{i}=Z_{i} \backslash\left\{U_{1, i} \cup U_{2, i}\right\}, \quad i=1,2, \ldots, n,
$$

where

$$
\begin{aligned}
& U_{1, i}=\left\{\left(z_{1}, z_{2}\right): z_{1} \in\left[z_{1, i}-\frac{z_{2}\left|z_{2}\right|}{2 \widetilde{V}_{i}}, z_{2}>0\right]\right\}, \\
& U_{2, i}=\left\{\left(z_{1}, z_{2}\right): z_{1} \in\left[-z_{1, i}-\frac{z_{2}\left|z_{2}\right|}{2 \widetilde{V}_{i}}, z_{2}<0\right]\right\} .
\end{aligned}
$$

Proof. Region $\bar{R}_{i}$ is the largest positively invariant set contained in $R_{i}$. The proof of this theorem can be found in [39].

Theorem 8. Consider the state-space partitioning (12) ad (13) and the region definition (17) and assume that the bounds (15) hold. Under the control of the proposed method, the auxiliary system states converge to the origin in finite time.

Proof. The time sequence $\left\{t_{\mathrm{Max}}\right\}$ is the time instant at which an extremal value of $z_{1}$ occurs, where

$$
\begin{aligned}
t_{\operatorname{Max}_{i+1}}= & t_{\mathrm{Max}_{i}} \\
+\frac{\left(\alpha_{i}^{*} g_{2, i}+g_{1, i}\right) V_{i}}{\left(g_{1, i} V_{i}-F_{i}\right) \sqrt{\left(\alpha_{i}^{*} g_{2, i} V_{i}+F_{i}\right)}} \sqrt{z_{\operatorname{Max}_{i}}} & \\
i & =1,2, \ldots, n .
\end{aligned}
$$

By means of simple computations, the following inequality holds:

$$
\begin{aligned}
t_{\mathrm{Max}_{i+1}} \leq & t_{\mathrm{Max}_{1}} \\
& +\max _{j=2,3, \ldots, n}\left(\frac{3 \sqrt{2}}{2 \sqrt{g_{1, i} V_{i}-F_{i}-H}}\right) \sum_{i=1}^{n} \sqrt{z_{\mathrm{Max}_{i}}} \\
= & \eta \sum_{i=1}^{n} \sqrt{z_{\mathrm{Max}_{i}}}+t_{\mathrm{Max}_{1}} .
\end{aligned}
$$

Then, inserting (26) into (23) yields

$$
\left|z_{\operatorname{Max}_{i}}\right| \leq\left|-\frac{\left(\alpha_{i}^{*} g_{2, i}-g_{1, i}\right) V_{i}+2\left(F_{i}+H\right)}{2\left(g_{1, i} V_{i}-F_{i}-H\right)}\right|^{i-1}\left|z_{\mathrm{Max}_{1}}\right| .
$$

From (31) and (32), it can be obtained that

$$
\begin{aligned}
& t_{\mathrm{Max}_{i+1}} \\
& \leq \eta \sum_{i=1}^{n}\left|-\frac{\left(\alpha_{i}^{*} g_{2, i}-g_{1, i}\right) V_{i}+2\left(F_{i}+H\right)}{2\left(g_{1, i} V_{i}-F_{i}-H\right)}\right|^{i-1} \sqrt{\left|z_{\mathrm{Max}_{1}}\right|} \\
& \quad+t_{\mathrm{Max}_{1}} \\
& =\eta^{\prime} \sum_{i=1}^{n}\left|-\frac{\left(\alpha_{i}^{*} g_{2, i}-g_{1, i}\right) V_{i}+2\left(F_{i}+H\right)}{2\left(g_{1, i} V_{i}-F_{i}-H\right)}\right|^{i-1}+t_{\mathrm{Max}_{1}} .
\end{aligned}
$$

Obviously, $\left|-\left(\left(\alpha_{i}^{*} g_{2, i}-g_{1, i}\right) V_{i}+2\left(F_{i}+H\right)\right) / 2\left(g_{1, i} V_{i}-F_{i}-H\right)\right|<$ 1 . Therefore, according to (31), there is

$$
\lim _{i \rightarrow \infty} z_{\mathrm{Max}_{i}}=0 .
$$

Finally,

$$
\begin{aligned}
& \lim _{i \rightarrow \infty} t_{\operatorname{Max}_{i}} \\
& <\frac{\eta^{\prime}}{1-\left|\left(\left(\alpha_{i}^{*} g_{2, i}-g_{1, i}\right) V_{i}+2\left(F_{i}+H\right)\right) / 2\left(g_{1, i} V_{i}-F_{i}-H\right)\right|} \\
& \quad+t_{\operatorname{Max}_{1} .}
\end{aligned}
$$

The convergence of the time sequence $\left\{z_{\mathrm{Max}}\right\}$ in finite time implies the convergence to zero of the auxiliary system. Then, the NWMR system will be steered to the desired trajectory in finite time. Here, the proof is complete.

\section{Simulations and Experimental Results}

In this section, simulation and experiment studies for tracking control of a NWMR system are conducted to verify the effectiveness of the proposed control laws. The numerical values of the parameters are listed in Table 1. 


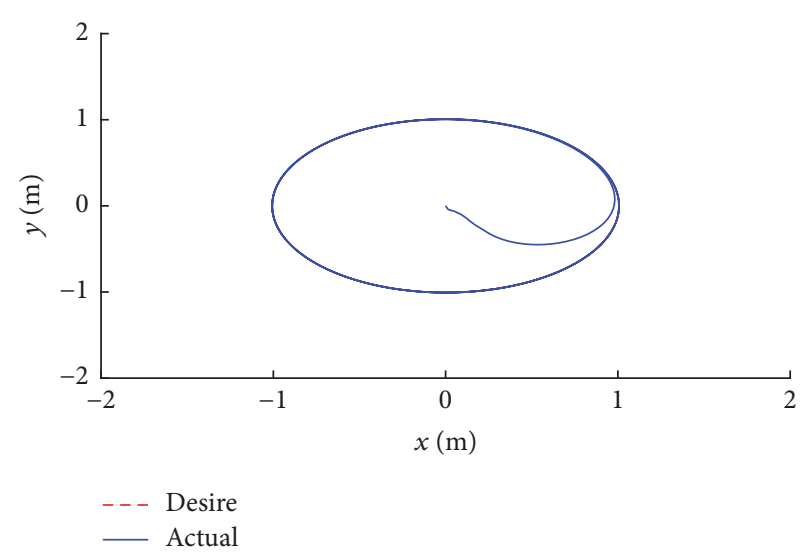

(a) Robot circular trajectory in $x-y$ plane

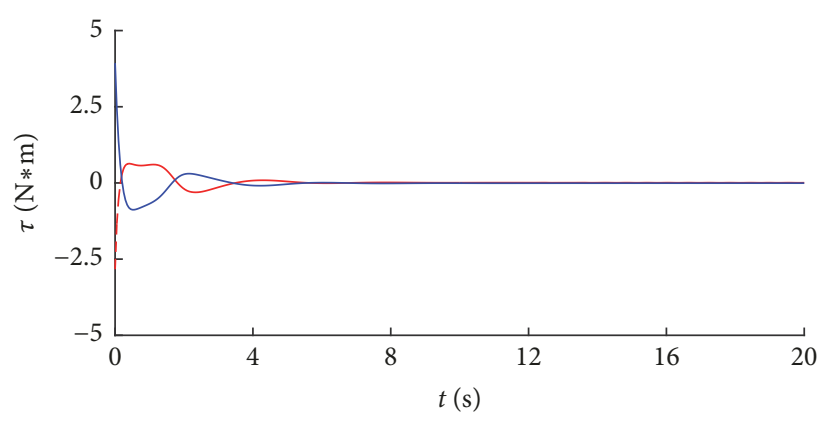

$---\tau_{\mathrm{r}}$

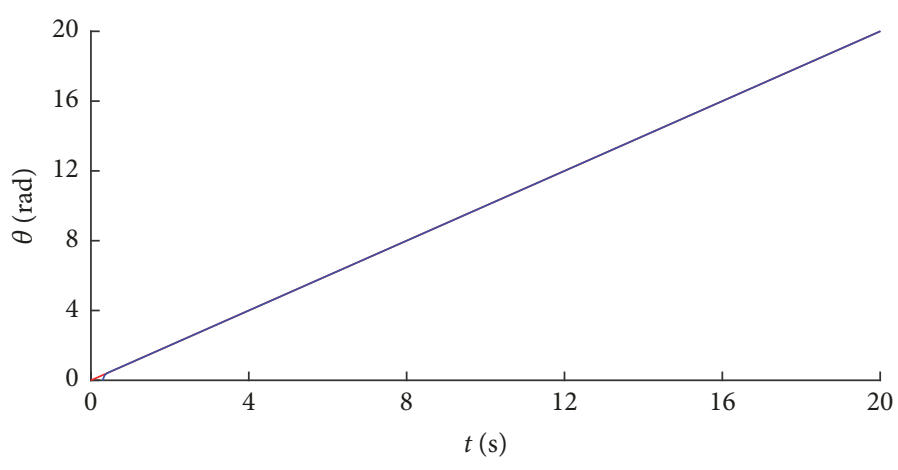

$---\theta_{r}$

$-\theta$

(b) The output $\theta$ trajectory

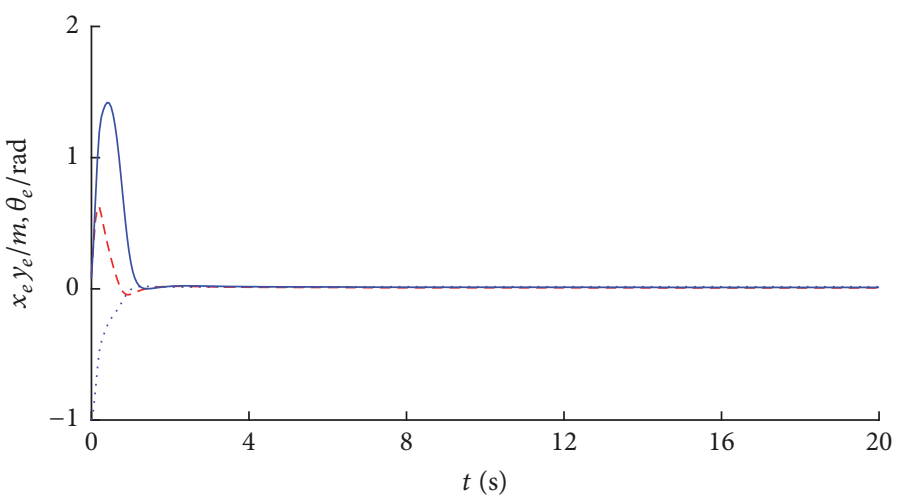

$\begin{aligned}--- & x_{e} \\ \ldots . . . & y_{e} \\ - & \theta_{e}\end{aligned}$ (c) Control torque $\tau$

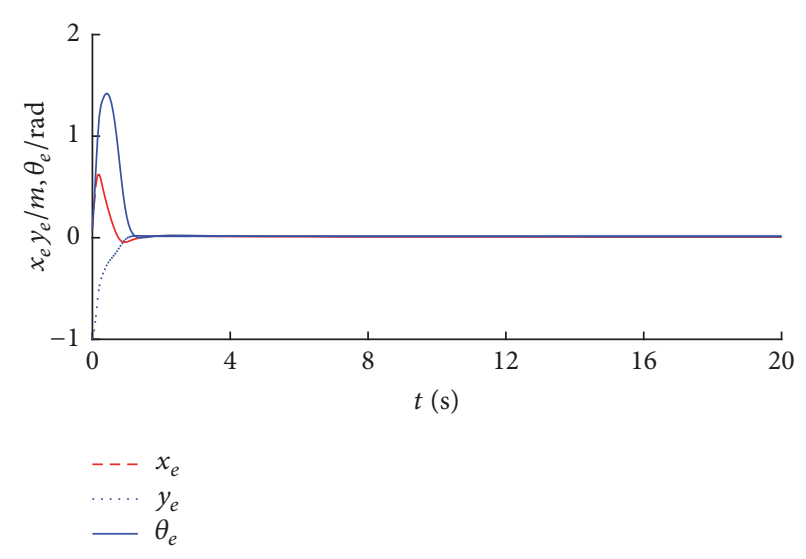

(e) Zoomed trajectory in $z_{1}-z_{2}$ plane (d) Overall trajectory in $z_{1}-z_{2}$ plane

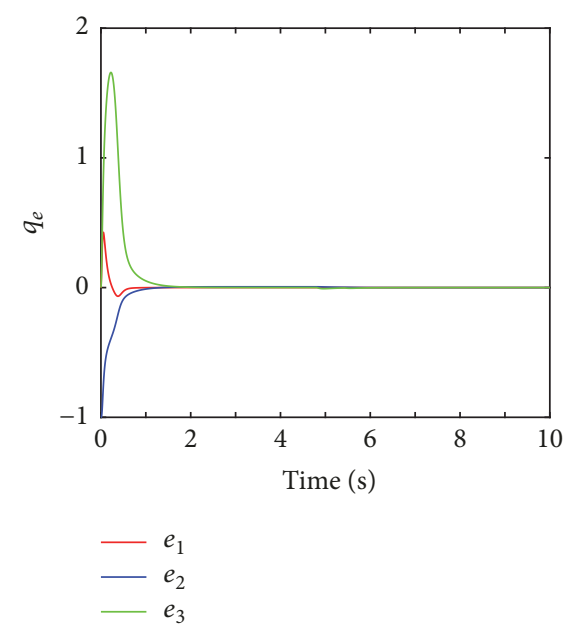

(f) The tracking errors $q_{e}$

FIgURE 3: Simulation results of the mobile robot with the proposed controller.

5.1. Simulations Results. The tracking controller is compared with the traditional sliding-mode controller, and the adjustment of $\alpha_{i}$ in Remark 6 is verified.

Compared with Figures 3(a) and 3(b) and Figures 4(a) and $4(\mathrm{~b})$, one can see that both trajectories can track the desired trajectory. However, it can be observed from Figure 3(f) that the tracking errors decreased to zero, which cost $1.5 \mathrm{~s}$ less than in Figure 4(d), which indicates that the proposed method has a better response with a faster convergence speed. In Figure 3(c), the torque control input 


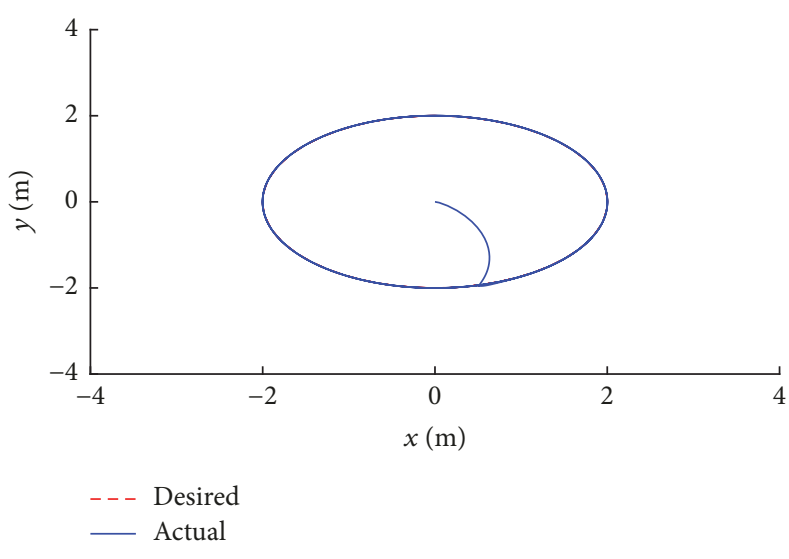

(a) Robot circular trajectory in $x-y$ plane

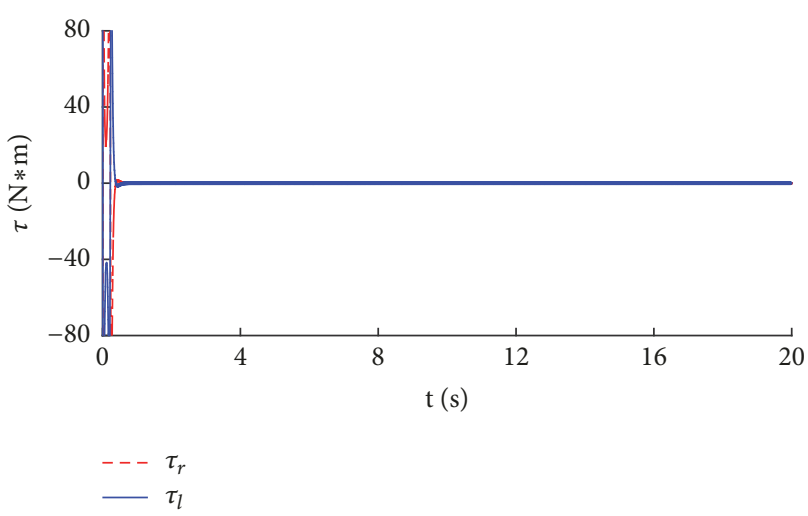

(c) Control torque $\tau$

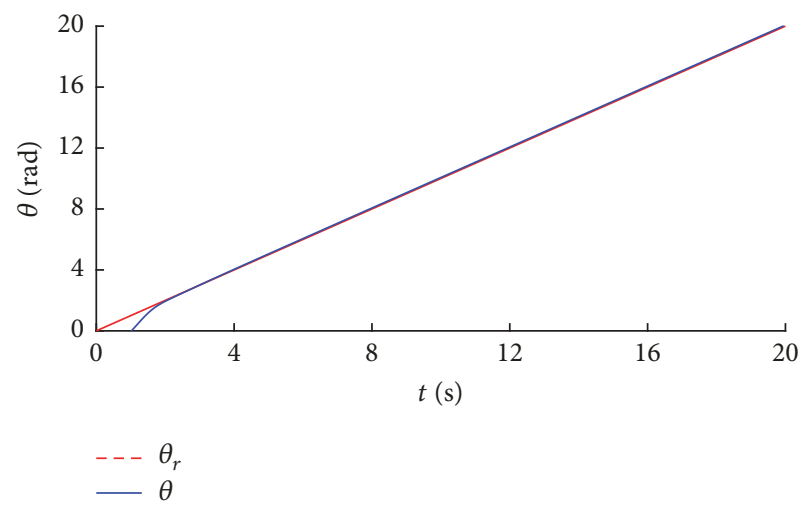

(b) The output $\theta$ trajectory

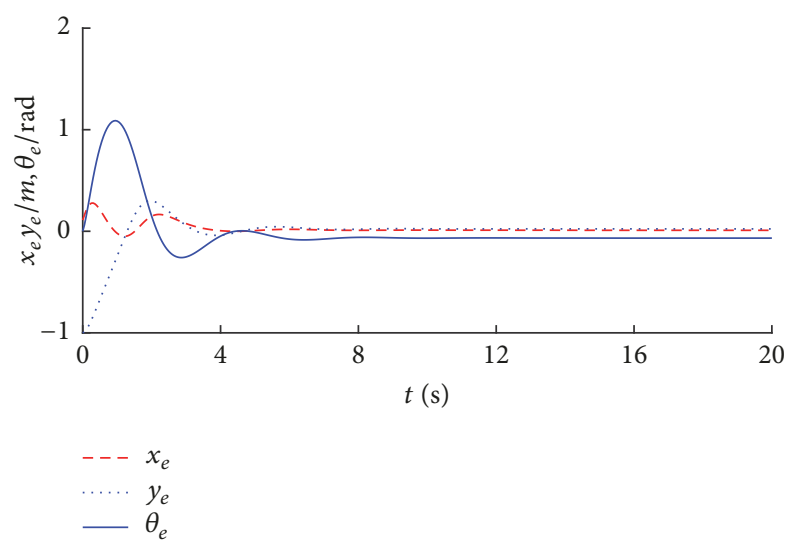

(d) The tracking errors $q_{e}$

FIGURE 4: Simulation results of the mobile robot with the SMC controller.

TABLE 1: Parameters used for the simulation.

\begin{tabular}{lc}
\hline$m=2.92 \mathrm{~kg}$ & $\alpha^{*}=[0.65,0.30,0.8]^{\mathrm{T}}$ \\
$d=0.11 \mathrm{~m}$ & {$\left[k_{1}, k_{2}, k_{3}\right]=[3,120,20]$} \\
$I=2.5 \mathrm{~kg} \mathrm{~m}^{2}$ & $z_{1}=[70,50,5 ; 70,50,5]^{\mathrm{T}}$ \\
$R=0.17 \mathrm{~m}$ & $z_{2}=[70,50,5 ; 70,50,5]^{\mathrm{T}}$ \\
$r=0.03 \mathrm{~m}$ & {$\left[v_{o 1 r}, \omega_{o 1 r}\right]=[1.0 \mathrm{~m} / \mathrm{s}, 1.0 \mathrm{rad} / \mathrm{s}]$} \\
$\lambda=6.9$ & $V=[5.0,1.80,1.0 ; 5.0,0.81,0.3]^{\mathrm{T}}$ \\
$H=0.3$ & $\bar{\tau}_{d}+\bar{F}(q, \dot{q}, \ddot{q})=[0.1 * \eta+0.1 \sin (t), 0.2 * \dot{\eta}+0.1 \cos (t)]^{\mathrm{T}}$ \\
\hline
\end{tabular}

$\tau$ of the proposed control can be confined to an arbitrarily small repose, and the chattering is well attenuated compared with that in Figure 4(c).

5.2. Experimental Results. Experiments are conducted to test the effectiveness of the proposed control laws in a practical NWMR system. QUANSER QBot2 as shown in Figure 5(a) is used for the experiments. Figure 5(b) shows the diagram of experimental platform. The robots move on a $300 \times 360 \mathrm{~cm}$ white surface. In the experiment, the robot starts at $x=0 \mathrm{~m}$, $y=0 \mathrm{~m}$, and $\theta=0 \mathrm{rad} / \mathrm{s}$ and should follow a circle trajectory of reference. The maximum velocity of QUANSER QBot2 is $v=0.7 \mathrm{~m} / \mathrm{s}$, so the reference velocities in experiment are chosen as $v_{o 1 r}=0.3 \mathrm{~m} / \mathrm{s}$ and $\omega_{o 1 r}=0.25 \mathrm{rad} / \mathrm{s}$ and the radius of the circle is $1.2 \mathrm{~m}$.

The experimental results shown in Figure 6 illustrate the trajectory tracking result for a circle trajectory. The actual trajectory reaches the desired circle quickly. According to the experimental results for the circle trajectory tracking, the trajectory can remain stable and robust despite external disturbances and unmodeled dynamics in the robot.

In summary, both simulation studies and the experiment results demonstrate that the proposed control laws are effective.

\section{Conclusion}

An innovative robust controller has been investigated for dealing with the tracking problem of NWMR. Finite-time convergence of tracking error was achieved in the presence of external disturbances and unmodeled dynamics. Comparing simulation and experimental studies, the effectiveness and the superior performance of the proposed control method are verified. There are several features that are worth pointing out. (1) The assumption of bound for the uncertain functions can be relaxed. (2) The NWMR can accommodate different control objectives under different requirements. (3) The chattering problem is effectively alleviated and has a better 


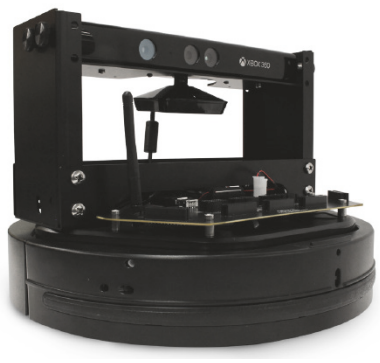

(a) QUANSER QBot2

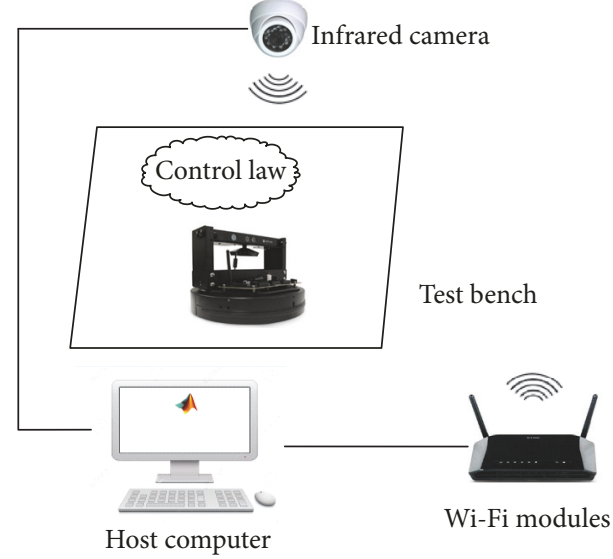

(b) Diagram of experiment platform

FIGURE 5: Experimental environment.

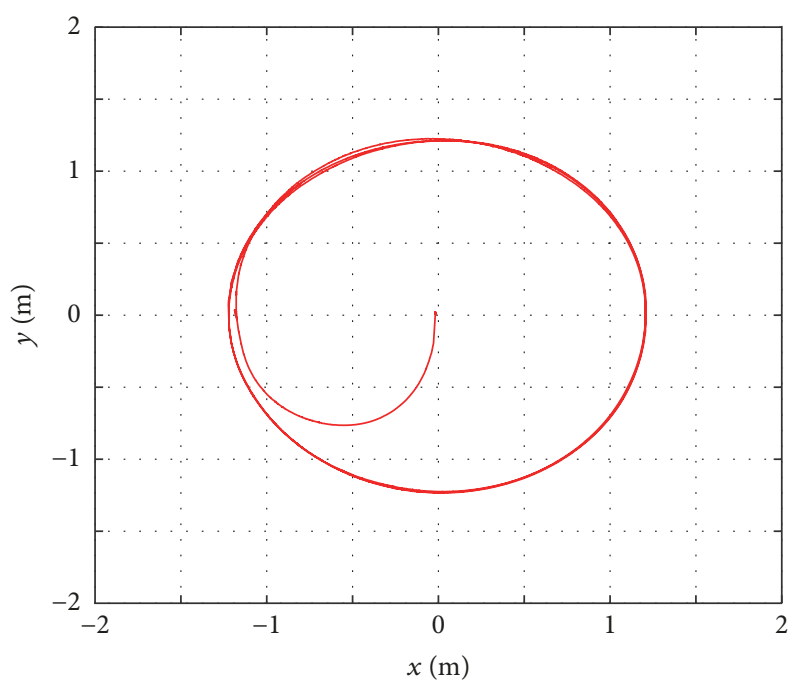

- Actual

(a) Robot circular trajectory in $x-y$ plane

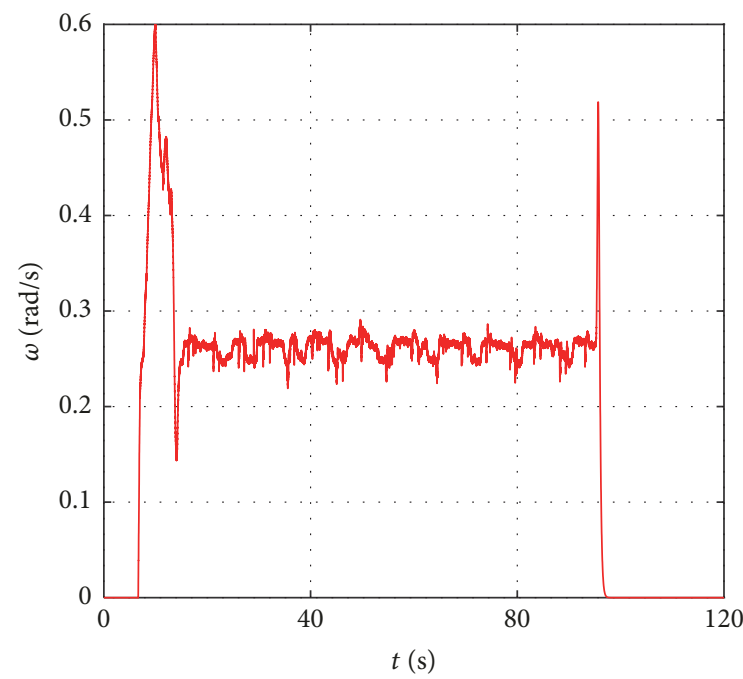

(c) The angular velocity $\omega$

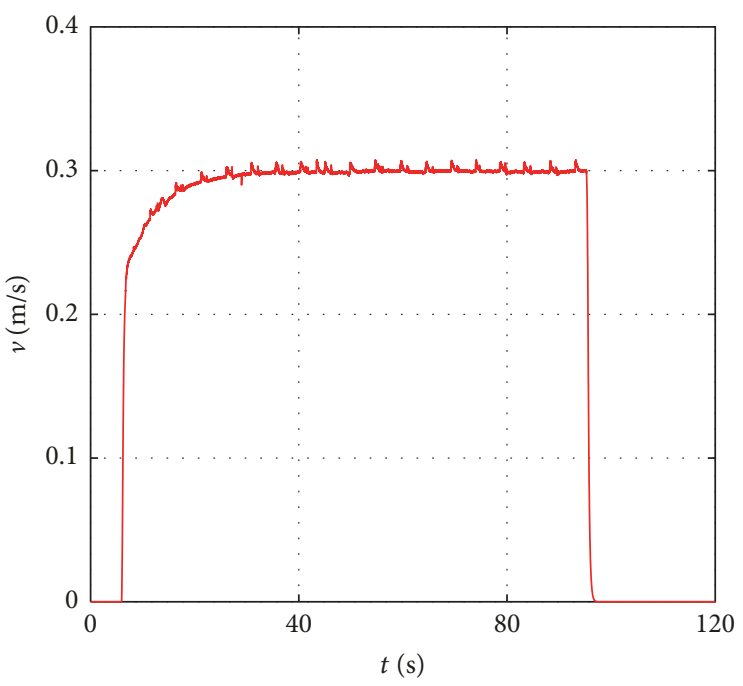

(b) The linear velocity $v$

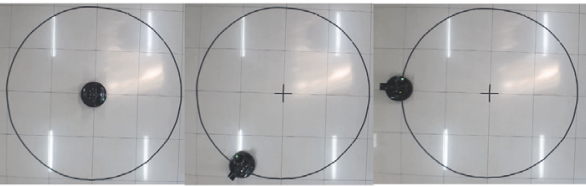

(d) The trajectories of the robot

FIGURE 6: Experiment results of the mobile robot with the proposed controller. 
response with a faster convergence speed. In future work, the assumption of the bound of disturbance should be relaxed so that this control algorithm can be applied to more nonlinear systems.

\section{Conflicts of Interest}

The authors declare that they have no conflicts of interest.

\section{Acknowledgments}

This work is supported by the NSFC under Grant 61473248 and by the Natural Science Foundation of Hebei Province under Grant F2016203496.

\section{References}

[1] K.-D. Nguyen and H. Dankowicz, "Adaptive control of underactuated robots with unmodeled dynamics," Robotics and Autonomous Systems, vol. 64, pp. 84-99, 2015.

[2] T.-C. Lee, "Exponential stabilization for nonlinear systems with applications to nonholonomic systems," Automatica, vol. 39, no. 6, pp. 1045-1051, 2003.

[3] K. Worthmann, M. W. Mehrez, M. Zanon, G. K. I. Mann, R. G. Gosine, and M. Diehl, "Model predictive control of nonholonomic mobile robots without stabilizing constraints and costs," IEEE Transactions on Control Systems Technology, vol. 24, no. 4, pp. 1394-1406, 2016.

[4] S. Yildirim and S. Savas, "Tracking control of a nonholonomic mobile robot using neural network," in Handbook of Research on Advancements in Robotics and Mechatronics, pp. 631-661, 2014.

[5] A. Al-Mayyahi, W. Wang, and P. Birch, "Design of fractionalorder controller for trajectory tracking control of a non-holonomic autonomous ground vehicle," Journal of Control, Automation and Electrical Systems, vol. 27, no. 1, pp. 29-42, 2016.

[6] Z. Peng, G. Wen, S. Yang, and A. Rahmani, "Distributed consensus-based formation control for nonholonomic wheeled mobile robots using adaptive neural network," Nonlinear Dynamics, vol. 86, no. 1, pp. 605-622, 2016.

[7] S. Yang, Y. Cao, Z. Peng, G. Wen, and K. Guo, "Distributed formation control of nonholonomic autonomous vehicle via RBF neural network," Mechanical Systems and Signal Processing, vol. 87, part B, pp. 81-95, 2017.

[8] M. Boukens and A. Boukabou, "Design of an intelligent optimal neural network-based tracking controller for nonholonomic mobile robot systems," Neurocomputing, vol. 226, pp. 46-57, 2017.

[9] Z. Peng, S. Yang, G. Wen, A. Rahmani, and Y. Yu, "Adaptive distributed formation control for multiple nonholonomic wheeled mobile robots," Neurocomputing, vol. 173, pp. 1485-1494, 2016.

[10] W. Liu, C. Lim, P. Shi, and S. Xu, "Backstepping fuzzy adaptive control for a class of quantized nonlinear systems," IEEE Transactions on Fuzzy Systems, vol. 25, no. 5, pp. 1090-1101, 2017.

[11] J. Ye, "Tracking control for nonholonomic mobile robots: Integrating the analog neural network into the backstepping technique," Neurocomputing, vol. 71, no. 16-18, pp. 3373-3378, 2008.

[12] B. Chen, H. Zhang, and C. Lin, "Observer-based adaptive neural network control for nonlinear systems in nonstrict-feedback form," IEEE Transactions on Neural Networks and Learning Systems, vol. 27, no. 1, pp. 89-98, 2016.
[13] V. Goyal, V. Deolia K, and T. Sharma, "Neural network based sliding mode control for uncertain discrete-time nonlinear systems with time-varying delay," International Journal of Computational Intelligence Research, vol. 12, no. 2, pp. 125-138, 2016.

[14] G. Lai, Z. Liu, Y. Zhang, X. Chen, and C. L. Chen, "Robust adaptive fuzzy control of nonlinear systems with unknown and timevarying saturation," Asian Journal of Control, vol. 17, no. 3, pp. 791-805, 2015.

[15] H. Gao, X. Song, L. Ding, K. Xia, N. Li, and Z. Deng, "Adaptive motion control of wheeled mobile robot with unknown slippage," International Journal of Control, vol. 87, no. 8, pp. 15131522, 2014.

[16] J. Huang, C. Wen, W. Wang, and Z.-P. Jiang, "Adaptive output feedback tracking control of a nonholonomic mobile robot," Automatica, vol. 50, no. 3, pp. 821-831, 2014.

[17] M. Sharifi and H. Moradi, "Nonlinear robust adaptive sliding mode control of influenza epidemic in the presence of uncertainty," Journal of Process Control, vol. 56, pp. 48-57, 2017.

[18] Q. H. Ngo, N. P. Nguyen, C. N. Nguyen, T. H. Tran, and Q. P. $\mathrm{Ha}$, "Fuzzy sliding mode control of an offshore container crane," Ocean Engineering, vol. 140, pp. 125-134, 2017.

[19] C.-L. Hwang and H.-M. Wu, "Trajectory tracking of a mobile robot with frictions and uncertainties using hierarchical sliding-mode under-actuated control," IET Control Theory \& Applications, vol. 7, no. 7, pp. 952-965, 2013.

[20] J. X. Xu, Z. Q. Guo, and T. H. Lee, "Design and implementation of integral sliding-mode control on an underactuated twowheeled mobile robot," IEEE Transactions on Industrial Electronics, vol. 61, no. 7, pp. 3671-3681, 2014.

[21] L. Xin, Q. Wang, J. She, and Y. Li, "Robust adaptive tracking control of wheeled mobile robot," Robotics and Autonomous Systems, vol. 78, pp. 36-48, 2016.

[22] Y.-J. Huang, T.-C. Kuo, and S.-H. Chang, "Adaptive slidingmode control for nonlinear systems with uncertain parameters," IEEE Transactions on Systems, Man, and Cybernetics, Part B: Cybernetics, vol. 38, no. 2, pp. 534-539, 2008.

[23] I. F. Jasim, P. W. Plapper, and H. Voos, "Adaptive sliding mode fuzzy control for unknown robots with arbitrarily-switched constraints," Mechatronics, vol. 30, pp. 174-186, 2015.

[24] D. Shen, Z. Sun, and Y. Qiao, "Second-order sliding mode control for nonholonomic mobile robots formation," in Proceedings of the 30th Chinese Control Conference, CCC 2011, pp. 48604864, chn, July 2011.

[25] Y. Kali, K. Benjelloun, A. Fatemi, M. Saad, and M. Benbrahim, "Second order sliding mode with time delay control for uncertain robot manipulators," in Proceedings of the 2016 IEEE International Multidisciplinary Conference on Engineering Technology, IMCET 2016, pp. 160-165, Lebanon, November 2016.

[26] X. Huang, Y. Yan, and Y. Zhou, "Neural network-based adaptive second order sliding mode control of Lorentz-augmented spacecraft formation," Neurocomputing, vol. 222, pp. 191-203, 2017.

[27] Y. Huangfu, R. Ma, and A. Miraoui, "Avoidance high-frequency chattering second-order sliding mode controller design: buck converter in wind power system," International Journal of Antennas and Propagation, vol. 2012, Article ID 176830, 5 pages, 2012.

[28] G. Bartolini, A. Ferrara, L. Giacomini, and E. Usai, "Properties of a combined adaptive/second-order sliding mode control algorithm for some classes of uncertain nonlinear systems," Institute of Electrical and Electronics Engineers Transactions on Automatic Control, vol. 45, no. 7, pp. 1334-1341, 2000. 
[29] E. S. El Youssef, N. A. Martins, E. R. De Pieri, and U. F. Moreno, "PD-super-twisting second order sliding mode tracking control for a nonholonomic wheeled mobile robot," in Proceedings of the 19th IFAC World Congress on International Federation of Automatic Control, IFAC 2014, pp. 3827-3832, zaf, August 2014.

[30] S. Mondal and C. Mahanta, "Adaptive second order terminal sliding mode controller for robotic manipulators," Journal of The Franklin Institute, vol. 351, no. 4, pp. 2356-2377, 2014.

[31] Y. Zhao, Y. Sheng, and X. Liu, "A novel finite time sliding mode control for robotic manipulators," in Proceedings of the 19th IFAC World Congress on International Federation of Automatic Control, IFAC 2014, vol. 47, pp. 7336-7341, zaf, August 2014.

[32] S. Matoba, N. Nakamura, H. Nakamura, and H. Nishitani, "Robust finite-time control of robot manipulators," in Proceedings of the 18th IFAC World Congress, vol. 44, pp. 11863-11868, Italy, September 2011.

[33] M. Tanelli and A. Ferrara, "Enhancing robustness and performance via switched second order sliding mode control," Institute of Electrical and Electronics Engineers Transactions on Automatic Control, vol. 58, no. 4, pp. 962-974, 2013.

[34] A. Pisano, M. Tanelli, and A. Ferrara, "Switched/time-based adaptation for second-order sliding mode control," Automatica, vol. 64, pp. 126-132, 2016.

[35] A. Saradagi, V. Muralidharan, V. Krishnan, S. Menta, and A. D. Mahindrakar, "Formation control and trajectory tracking of nonholonomic mobile robots," IEEE Transactions on Control Systems Technology, vol. 99, pp. 1-9, 2017.

[36] Y. Kanayama, Y. Kimura, F. Miyazaki, and T. Noguchi, "A stable tracking control method for an autonomous mobile robot," in Proceedings of the IEEE International Conference on Robotics and Automation, vol. 1, pp. 384-389, Washington, DC, USA, May 1990.

[37] B. Sharma, J. Vanualailai, and S. Singh, “Tunnel passing maneuvers of prescribed formations," International Journal of Robust and Nonlinear Control, vol. 24, no. 5, pp. 876-901, 2014.

[38] B. Sharma, J. Vanualailai, and S. Singh, "Motion planning and posture control of multiple $n$-link doubly nonholonomic manipulators," Robotica, vol. 35, no. 1, pp. 1-25, 2017.

[39] M. Rubagotti and A. Ferrara, "Second order sliding mode control of a perturbed double integrator with state constraints," in Proceedings of the 2010 American Control Conference, ACC 2010, pp. 985-990, usa, July 2010. 


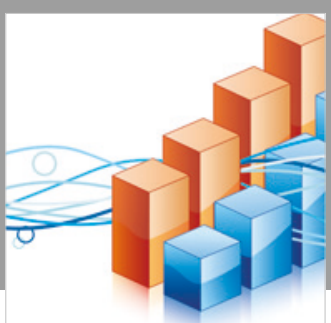

Advances in

Operations Research

\section{-n-m}
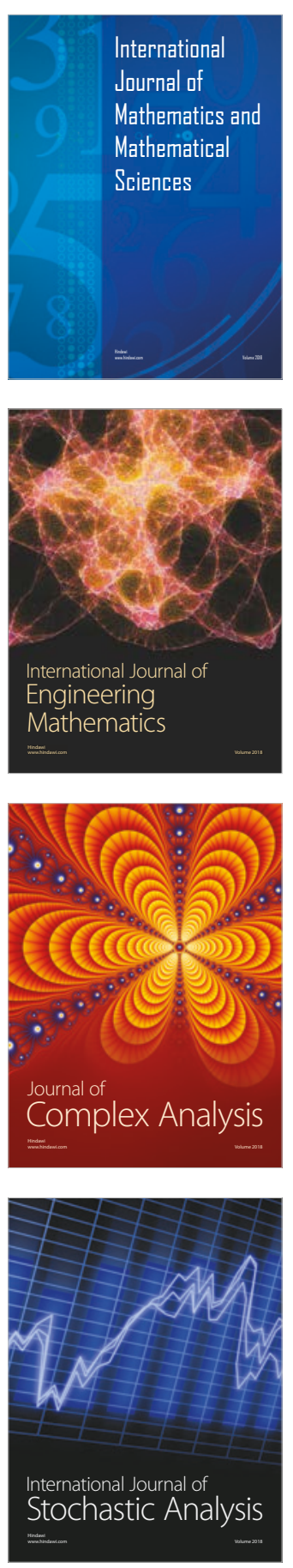
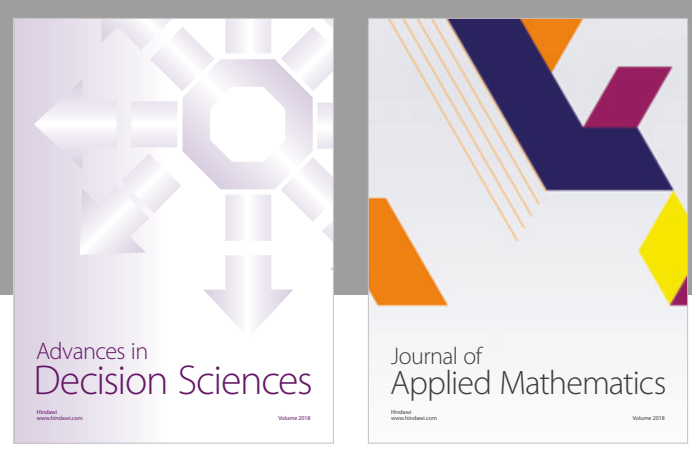

Journal of

Applied Mathematics
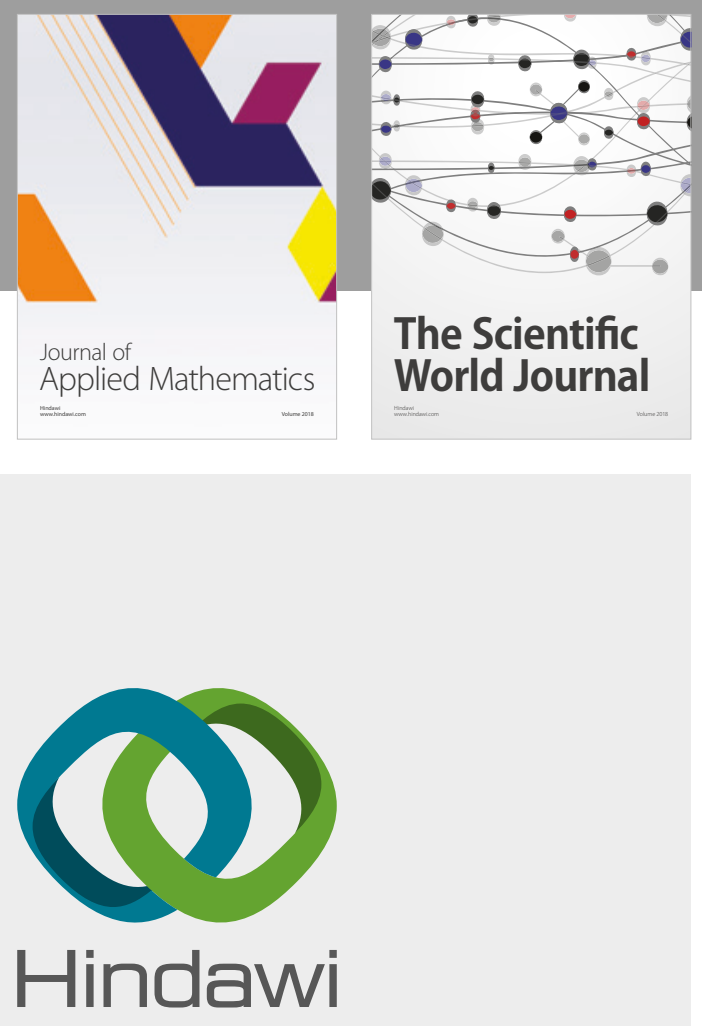

Submit your manuscripts at

www.hindawi.com

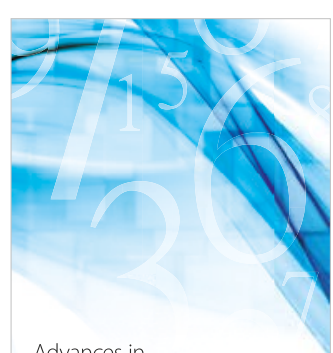

Advances in
Numerical Analysis
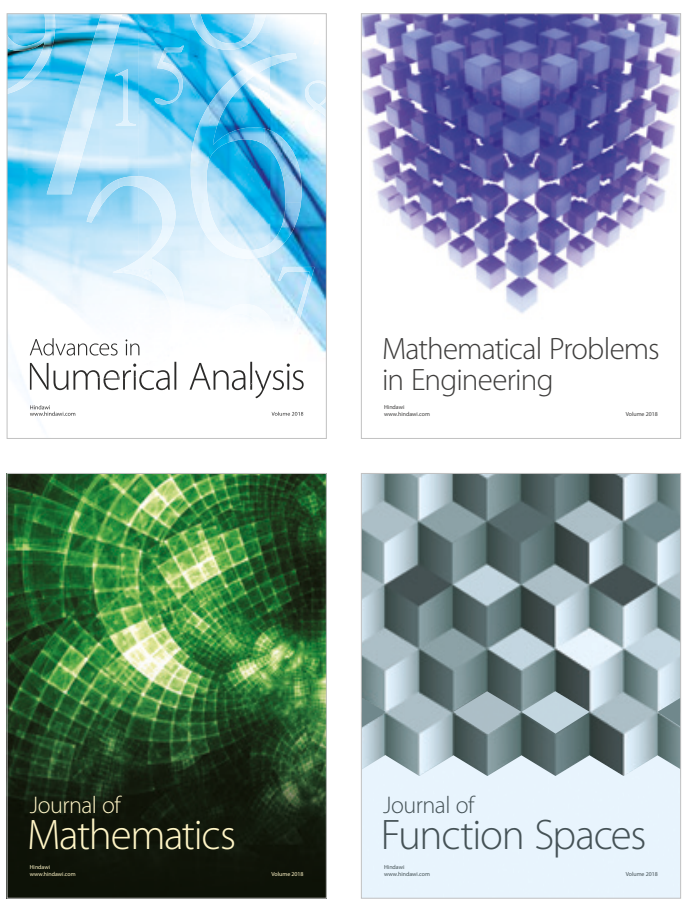

Mathematical Problems in Engineering

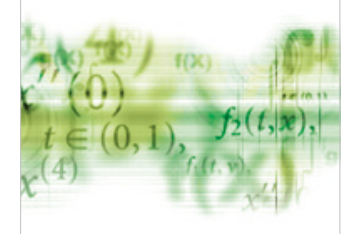

International Journal of

Differential Equations

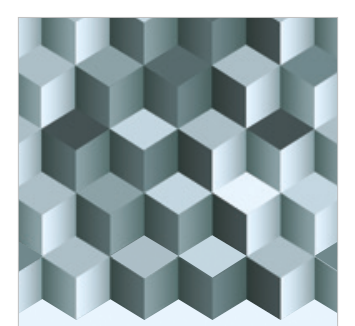

Journal of

Function Spaces
The Scientific

World Journal

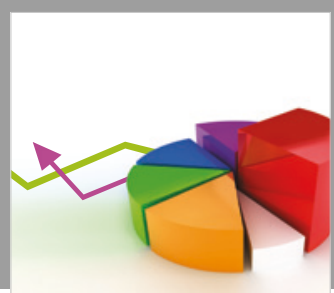

Journal of

Probability and Statistics
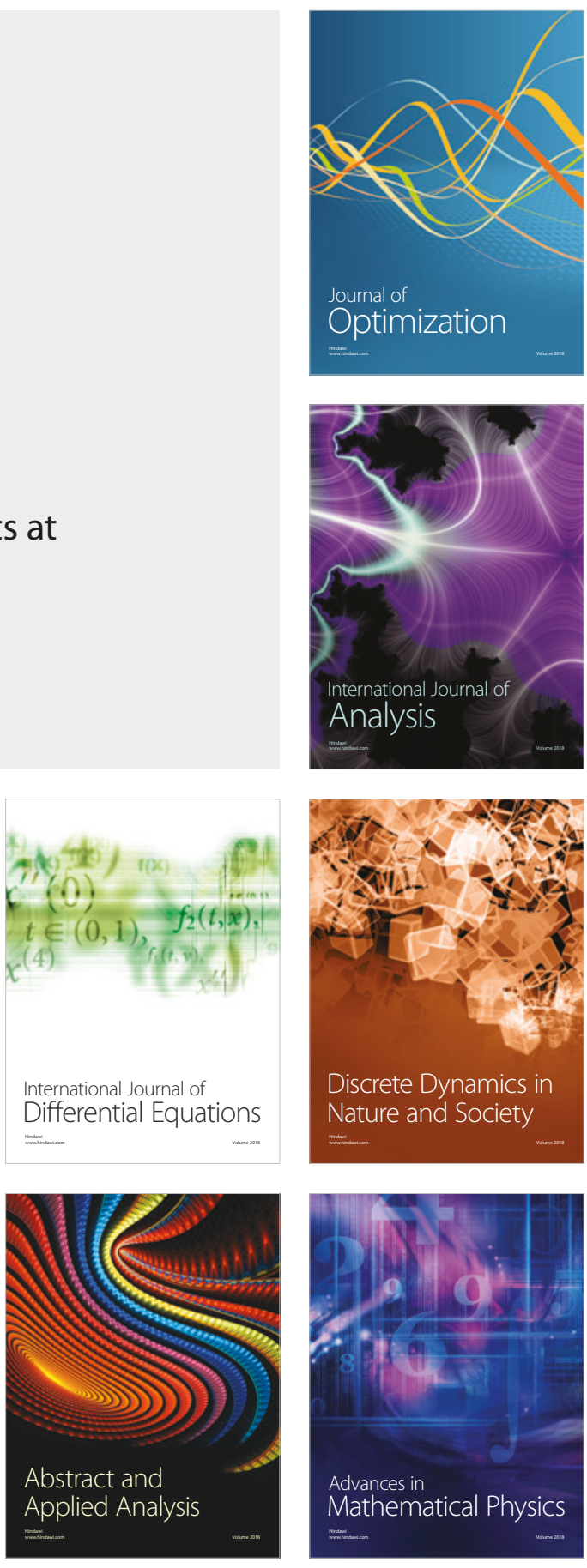